\title{
Spin energy enhanced by exchange interaction in a Rashba spintronic system in quantizing magnetic fields
}

\author{
C. H. Yang ${ }^{\text {a) }}$ \\ Nanjing University of Information Science and Technology, Nanjing 219944, China \\ W. $\mathrm{Xu}^{\mathrm{b})}$ \\ Institute of Solid State Physics, Chinese Academy of Sciences, Hefei 230031, China \\ and Department of Theoretical Physics, Research School of Physical Sciences and Engineering, \\ Australian National University, Canberra ACT 0200, Australia
}

(Received 26 September 2007; accepted 2 November 2007; published online 14 January 2008)

\begin{abstract}
Using the standard Hartree-Fock approximation on the basis of the random-phase approximation, we calculate the self-energy induced by exchange scattering due to electron-electron interaction in a spin-polarized two-dimensional electron gas (2DEG) system. Applying the exchange self-energy to a Green's function approach, we examine how the exchange interaction affects the spin-splitting in a 2DEG with the Rashba spin-orbit interaction and Zeeman spin-splitting in the presence of quantizing magnetic fields. It is demonstrated that such a many-body interaction can lower the energy levels of the system and enhance significantly the spin-splitting around the Fermi level. We also find that the exchange-enhanced spin-splitting is achieved mainly via intra-Landau-level scattering channels and the spin energy oscillates with the magnetic fields. (c) 2008 American Institute of Physics. [DOI: 10.1063/1.2828175]
\end{abstract}

\section{INTRODUCTION}

In recent years, the investigation of two-dimensional electron gas (2DEG) systems with the Rashba spin-orbit interaction (RSOI) has become an important field of research in condensed matter physics and semiconductor electronics. The spin-polarized electronic (or spintronic) systems have been proposed as advanced electronic devices to be used in future quantum communication and information. It is known that a spin-polarized 2DEG can be realized on the basis of narrow-gap semiconductor heterojunctions or quantum wells, such as those realized from InAlAs/InGaAs heterostructures. ${ }^{1}$ When such systems are subjected to static magnetic fields, a strong RSOI can be observed on top of the Zeeman spin-splitting, due to the inversion asymmetry of the microscopic confining potential achieved by the presence of the heterojunction. ${ }^{2}$ At present, the magnetotransport measurement via Shubnikov-de Haas $(\mathrm{SdH})$ oscillations is one of the most popularly used experimental techniques to identify the RSOI in InGaAs-based spintronic systems. ${ }^{3}$ From the periodicity of the $\mathrm{SdH}$ oscillations, the electron densities in different spin branches can be determined and, from these results, the Rashba parameter of the system can be obtained. The Rashba parameter $\alpha$ is an important coefficient that measures the strength of the RSOI in a spintronic device. It was found experimentally that the Rashba parameter in InGaAsbased spintronic systems varies from $\alpha \sim 5 \times 10^{-12} \mathrm{eV}$ m in Ref. 5 to $\alpha \sim 10^{-11} \mathrm{eV} \mathrm{m}$ in Ref. 4 and to $\alpha \sim(3-4)$ $\times 10^{-11} \mathrm{eV}$ m in Ref. 3 .

Theoretically, the Rashba parameter can be calculated on the basis of the $\mathbf{k} \cdot \mathbf{p}$ band-structure calculations ${ }^{4}$ and selfconsistent calculations on the basis of the $\mathbf{k} \cdot \mathbf{p}$ results. ${ }^{5} \mathrm{Al}-$

\footnotetext{
${ }^{a)}$ Electronic mail: yangcuihong1978@163.com.

b) Electronic mail: wen105@rsphysse.anu.edu.au.
}

though in some cases a good agreement between experimental and theoretical results for the Rashba parameter can be reached (e.g., in Ref. 4), it is also found that $\alpha$ obtained theoretically is much smaller (at least a factor of 2 in Refs. 3 and 5) than those measured experimentally. It should be noted that the $\mathbf{k} \cdot \mathbf{p}$ band-structure calculation is basically a single-particle approach. In the self-consistent calculation using the $\mathbf{k} \cdot \mathbf{p}$ results, only the Hartree potential along the growth direction is considered and, therefore, such a calculation cannot include fully the many-body effects in the system. Recently published experimental results ${ }^{6}$ have indicated that the electron-electron (e-e) interaction can play an important role in spin-splitting in the Rashba spintronic systems. Hence, it is important and necessary to investigate the manybody effects in spin-split 2DEG systems in the presence of quantizing magnetic fields, and this is the prime motivation of the present theoretical work.

It is well known that in an electron gas system, manybody effect such as the exchange scattering induced by e-e interaction and electron interactions with scattering centers can enhance the spin-splitting in the device system. ${ }^{7}$ The exchange-enhanced spin-splitting in a 2DEG with the RSOI was studied successfully by Chen and Raikh ${ }^{8}$ using a Fermi liquid theory. In their work, the effect of Landau quantization was not included. They found that for the case of a weak magnetic field, the exchange interaction affects more strongly the Zeeman splitting than the Rashba splitting. It is known that in the presence of quantizing magnetic fields, a much stronger exchange interaction can be achieved because in such a case the Fermi level can be in between the energy levels with different spin orientations. Theoretical work has demonstrated previously that in the absence of the RSOI, the spin-splitting (induced by the Zeeman effect alone) in a 2DEG in the presence of quantizing magnetic fields can be 
enhanced significantly by the many-body effect such as exchange interaction induced by e-e interaction ${ }^{9}$ and/or by electron interactions with impurities and phonons. ${ }^{10}$ In this study, we generalize these well developed theoretical approaches to the case of a 2DEG with the RSOI. When a quantizing magnetic field is applied to a $2 \mathrm{DEG}$ with the RSOI, the Landau level (LL) structure has some unique features such as the LL mixing and shifting. Thus, the exchange scattering via e-e interaction in a spin-polarized $2 \mathrm{DEG}$ in the presence of quantizing magnetic fields can have some features different from those observed in a spin-degenerate $2 \mathrm{DEG}$ or in the absence of the quantizing magnetic fields. In this study, we apply the standard Hartree-Fock approximation and Green's function approach to examine the effect of the exchange interaction via e-e interaction on the energy spectrum of a spin-polarized 2DEG. We intend to examine how spin-splitting in a Rashba spintronic system is affected by the many-body effects in the presence of quantizing magnetic fields. The article is organized as follows. The theoretical approach of this study is developed in Sec. II. The numerical results are presented and discussed in Sec. III and the main conclusions obtained from the present study are summarized in Sec. IV.

\section{THEORETICAL APPROACH}

Here we consider a 2DEG formed in the $x y$ plane and a static magnetic field with a strength $B$ is applied along the $z$ axis (growth direction of the 2DEG). Including the Zeeman spin-splitting and the lowest-order of the RSOI obtained from a $\mathbf{k} \cdot \mathbf{p}$ band-structure calculation, the corresponding Schrödinger equation in the absence of e-e interaction can be solved analytically ${ }^{11}$ and the corresponding electron wave function and energy spectrum are obtained, respectively, as

$$
\Psi_{\lambda}(\mathbf{R})=e^{i k_{y} y} \psi_{n}(z)\left[\phi_{N}(X) \cos \theta_{N}^{s},-\phi_{N-1}(X) \sin \theta_{N}^{s}\right]
$$

in the form of a row matrix, and

$$
E_{\lambda}=E_{N, s}+\varepsilon_{n}=N \hbar \omega_{c}+s \hbar \Omega_{N} / 2+\varepsilon_{n},
$$

where the Landau gauge is taken for the vector potential of the magnetic field, $\mathbf{R}=(x, y, z), \lambda=\left(N, k_{y}, n, s\right)$ refers to quantum numbers, $N$ is the LL index, $k_{y}$ is a good quantum number along the $y$ direction, $s= \pm 1$ refers to the up or down spin state, $X=\left(x-x_{0}\right) / l_{B}$ with $l_{B}=(\hbar / e B)^{1 / 2}$ being the radius of the ground cyclotron orbit and $x_{0}=k_{y} l_{B}^{2}, \quad \hbar \Omega_{N}$ $=\sqrt{\left(\hbar \omega_{c}-E_{Z}\right)^{2}+8 N\left(\alpha / l_{B}\right)^{2}}$ with $E_{Z}=g \mu_{B} B$ being the energy of Zeeman splitting and $g$ the bare $g$ factor, $\alpha$ is the Rashba parameter, $\theta_{N}^{s}=\theta_{N^{+}}+(1-s) \pi / 4$ with $\operatorname{tg} \theta_{N}=\sqrt{8 N}\left(\alpha / l_{B}\right) /\left(\hbar \omega_{c}\right.$ $\left.-E_{Z}+\hbar \Omega_{N}\right), \omega_{c}=e B / m^{*}$ is the cyclotron frequency with $m^{*}$ being the electron effective mass, and $\phi_{N}(x)$ $=\left(\sqrt{\pi} l_{B} 2^{N} N !\right)^{-1 / 2} e^{-x^{2} / 2} H_{N}(x)$ with $H_{N}(x)$ being the Hermite polynomials. Moreover, the wave function $\psi_{n}(z)$ and electronic subband energy $\varepsilon_{n}$ are determined by a Schrödinger equation along the growth direction, which is independent of spin and magnetic field applied in this configuration. For the case of $N=0$, only the $s=+1$ state exists and the lowest energy level of the system is $E_{0,+1}=\hbar \omega_{c} / 2-E_{Z} / 2$ with a wave function $\Psi_{0, k_{v}, n,+1}(\mathbf{R})=e^{i k_{y} y} \psi_{n}(z)\left[\phi_{0}(X), 0\right]$. These results indicate that in the presence of RSOI, LL shifting and mixing can occur. We note that the wave function shown in Eq. (1) looks much simpler than those obtained previously. ${ }^{11}$

With the single-electron wave function and corresponding electronic energy spectrum, we can calculate the electrostatic energy induced by bare e-e interaction and the electron density-density correlation function (or pair bubble). On the basis of these results, we can calculate the effective e-e interaction and derive the dielectric function matrix induced by e-e interaction using the standard random-phase approximation (RPA). ${ }^{12}$ For a strongly confined 2DEG in which only the lowest electronic subband is occupied by electrons, we take $n=0$ and measure the energy from $\varepsilon_{0}=0$. The RPA dynamical dielectric function is then obtained as

$$
\varepsilon(\Omega, q)=1-V_{q} F(q) \Pi(\Omega, q),
$$

where $\Omega$ is the excitation frequency, $\mathbf{q}=\left(q_{x}, q_{y}\right)$ is the change of electron wave vector during an e-e scattering event, $V_{q}$ $=2 \pi e^{2} / \kappa q$ with $\kappa$ being the dielectric constant of the material, $F(q)=\int d z_{1} \int d z_{2}\left|\psi_{0}\left(z_{1}\right)\right|^{2}\left|\psi_{0}\left(z_{2}\right)\right|^{2} e^{-q\left|z_{1}-z_{2}\right|}$ is a form factor for e-e interaction along the growth direction in a $2 \mathrm{DEG}$, and

$$
\begin{aligned}
& \Pi(\Omega, q)=\frac{1}{2 \pi l_{B}^{2}} \sum_{N^{\prime}, N, s^{\prime}, s} C_{N^{\prime} N}^{s^{\prime} s}\left(l_{B}^{2} q^{2} / 2\right) \\
& \times \frac{f\left(E_{N^{\prime}, s^{\prime}}\right)-f\left(E_{N, s}\right)}{\hbar \Omega+E_{N^{\prime}, s^{\prime}}-E_{N, s}+i \delta}
\end{aligned}
$$

is the density-density correlation function with $f(x)$ being the Fermi-Dirac function. Moreover, $C_{N^{\prime} N}^{s^{\prime} s}(u)=[M ! /(M$ $+J) !] e^{-u} u^{J} I_{N^{\prime}, N}^{s^{\prime}, s}(u)$ is the form factor for e-e interaction in the 2D plane of a 2DEG, $M=\min \left(N^{\prime}, N\right), J=\left|N^{\prime}-N\right|$, and $I_{N^{\prime}, N}^{s^{\prime}, s}(u)=\left[L_{M}^{J}(u) \cos \theta_{N^{\prime}}^{s^{\prime}} \cos \theta_{N}^{s}+\left(1-\delta_{M, 0}\right) \sqrt{(M+J) / M} L_{M-1}^{J}\right.$ $\left.\times(u) \sin \theta_{N^{\prime}}^{s^{\prime}} \sin \theta_{N}^{s}\right]^{2}$ with $L_{N}^{J}(x)$ being the Laguerre polynomials. In a narrow-gap semiconductor heterostructure such as that realized from an InAlAs/InGaAs heterojunction, because of small electron effective mass $\left(\mathrm{m}^{*} / \mathrm{m}_{e} \sim 0.042\right)$, large dielectric constant $(\kappa \sim 13)$, and relatively high electron density $\left(n_{e} \sim 5 \times 10^{11} \mathrm{~cm}^{-2}\right)$, the interaction parameter $r_{s}$ $=e^{2} m^{*} /\left(4 \pi \kappa \hbar^{2} \sqrt{\pi n_{e}}\right) \sim 0.03 \ll 1$ can often be satisfied. Hence, for an InAlAs/InGaAs-based spintronic system, the usual RPA approximation can be employed to evaluate the dielectric response induced by e-e interaction.

Using the single-electron wave function, given by Eq. (1) to form the two-particle Slater wave function, the electrostatic scattering energy induced by e-e interaction via the Coulomb potential can be calculated on the basis of the Hartree-Fock approximation. ${ }^{13}$ In doing so, we can obtain the self-energy induced by bare e-e interaction $\mathcal{E}_{s s^{\prime}}(N)$ via Hartree $(\mathrm{H})$ and exchange (ex) or Fock scattering events: $\mathcal{E}_{s s^{\prime}}(N)=\mathcal{E}_{s s^{\prime}}^{\mathrm{H}}(N)+\mathcal{E}_{s s^{\prime}}^{\mathrm{ex}}(N)$. With the RPA dielectric function given by Eq. (3), the self-energy in the presence of e-e screening can then be calculated through $\Sigma_{s}^{s^{\prime}}(N)$ $=\epsilon^{-1}(q) \mathcal{E}_{s s^{\prime}}(N)$, where $\epsilon(q)=\lim _{\Omega \rightarrow o} \epsilon(\Omega, q)$ is the static dielectric function. Thus, in the presence of e-e screening, the self-energy induced by the Hartree scattering provides only a constant energy background, ${ }^{14}$ and the self-energy induced by exchange scattering via e-e interaction is obtained as 


$$
\Sigma_{s}^{s^{\prime}}(N)=-\sum_{N^{\prime}, \mathbf{q}} \frac{2 \pi e^{2}}{\kappa q-2 \pi e^{2} F(q) \Pi(q)} C_{N, N^{\prime}}^{s, s^{\prime}}\left(\frac{l_{B}^{2} q^{2}}{2}\right),
$$

with $\Pi(q)=\lim _{\Omega \rightarrow 0} \Pi(\Omega, q)$ being the static density-density correlation function.

Applying the self-energy induced by the exchange interaction to the diagrammatic techniques, ${ }^{13}$ the Green's function for a spin-polarized 2DEG in the presence of the RSOI and quantizing magnetic field can be written as

$$
G_{N}^{s s^{\prime}}(E)=\left[G_{N s}^{-1}(E)-\Sigma_{s}^{s^{\prime}}(N)\right]^{-1},
$$

where $E$ is the electron energy, $G_{N S}(E)=\left(E-E_{N, S}+i \delta\right)^{-1}$ is the diagonal Green's function for a spin-split 2DEG in the absence of e-e interaction, and the off-diagonal components are induced by exchange self-energy due to e-e interaction. In the form of a matrix, we have

$$
G_{N}^{s s^{\prime}}(E)=\frac{1}{\Delta_{0}+i \Delta_{1} \delta}\left[\begin{array}{cc}
E_{-}(N)+i \delta & -\Sigma_{+}^{-}(N) \\
-\Sigma_{-}^{+}(N) & E_{+}(N)+i \delta
\end{array}\right] .
$$

Here, $E_{ \pm}(N)=E-E_{N, \pm}-\Sigma_{ \pm}^{ \pm}, \quad \Delta_{0}=E_{+}(N) E_{-}(N)-\Sigma_{+}^{-}(N) \Sigma_{-}^{+}(N)$, and $\Delta_{1}=E_{+}(N)+E_{-}(N)$. Thus, we obtain a live many-body quasiparticle with a lifetime $\tau_{N}=1 / \delta=\infty$ and the effective energy spectrum determined by $\Delta_{0}=0$, which reads

$$
E_{N, s}^{*}=N \hbar \omega_{c}+A_{N}^{+}+s \sqrt{\left(\hbar \Omega_{N} / 2+A_{N}^{-}\right)^{2}+\Delta^{2}},
$$

with $A_{N}^{ \pm}=\left[\Sigma_{+}^{+}(N) \pm \Sigma_{-}^{-}(N)\right] / 2, \Delta^{2}=\Sigma_{-}^{+}(N) \Sigma_{+}^{-}(N)$, and, again, $s= \pm 1$. Comparing $E_{N, s}^{*}$ with $E_{N, s}$, we see immediately that $E_{N, s}^{*}$ in the presence of e-e interaction is always smaller than $E_{N, s}$ because the exchange self-energy $\Sigma_{s}^{s^{\prime}}(N)<0$. When e-e interaction is considered, an energy-gap $\Delta$ is present within the effective energy spectrum. This energy-gap enhances the energy difference between two spin branches at a fixed LL index $N$.

\section{NUMERICAL RESULTS AND DISCUSSIONS}

In the numerical calculation, we take the typical sample and material parameters for a spin-polarized 2DEG based on an InAlAs/InGaAs heterostructure. For InGaAs, the electron effective mass is $m^{*}=0.042 m_{e}$, with $m_{e}$ being the rest electron mass, and the static dielectric constant is $\kappa=12.9$. In such systems, the electron density $n_{e} \sim 5 \times 10^{11} \mathrm{~cm}^{-2}$ and the Rashba parameter $\alpha \sim 1.5 \times 10^{-11} \mathrm{eV} \mathrm{m}$ obtained experimentally. ${ }^{3}$ In the calculation, the filling of electrons to the energy levels is determined by the condition of electron number conservation, which reads for the filling factor $\nu$ $=2 \pi l_{B}^{2} n_{e}=\Sigma_{N, f} f\left(E_{N, s}\right)$. In the present study, we limit ourselves to the case of low temperatures (i.e., $T \rightarrow 0$ ) where the strongest many-body effect, especially the exchange interaction, can be observed.

In Fig. 1, we show energy levels for a 2DEG subjected to a quantizing magnetic field in different situations: (I) only the Zeeman splitting is present where the Rashba parameter $\alpha=0$; (II) both the Zeeman splitting and the RSOI are included; and (III) the Zeeman splitting, the RSOI, and the exchange interaction are all considered. In the presence of the RSOI, LL mixing and shifting can occur (see II in Fig. 1) and these effects are more pronounced with increasing the

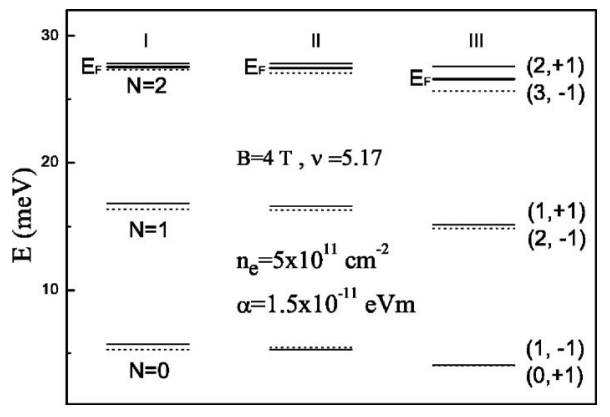

FIG. 1. Energy levels in the presence of (I) the Zeeman splitting alone (i.e., $\alpha=0$ ); (II) the Zeeman splitting and RSOI; and (III) the Zeeman splitting, RSOI, and exchange interaction at the fixed electron density $n_{e}$, magnetic field $B$, and Rashba parameter $\alpha$ as indicated. Here, $\nu$ is the filling factor, $E_{F}$ is the Fermi energy (thick solid lines), and the solid (dotted) lines are for spin-up (-down) states. The energy level $(N, s)$ corresponds to an energy $E_{N, s}$ in II and $E_{N, s}^{*}$ in III.

Rashba parameter $\alpha$. When e-e interaction is included (see III), the energy levels below the Fermi energy are lowered, which is consistent with the well known consequence that the exchange interaction lowers the energy of an electronic system. ${ }^{14}$ For the case $\nu=5.17$, the Fermi energy is in between the $(2,+1)$ and $(3,-1)$ states. As a result, a pronounced enhancement of the energy difference between spin-up and spin-down states can be observed around the Fermi level for the case of III, which implies an enhanced spin-splitting in the system by exchange interaction.

To see more clearly the enhancement of spin-splitting by exchange interaction in a $2 \mathrm{DEG}$ in the presence of the RSOI, the spin energy is shown in Fig. 2 as a function of magnetic field at the fixed Rashba parameter $\alpha$ and electron density $n_{e}$ for cases with and without inclusion of e-e interaction. Due to LL mixing and shifting by the RSOI (see Fig. 1), the spin energy around the Fermi level is defined as $E_{s}=\mid E_{N,+1}$ $-E_{N+1,-1} \mid$ or $E_{s}^{*}=\left|E_{N,+1}^{*}-E_{N+1,-1}^{*}\right|$ for the case of the absence or presence of e-e interaction, respectively. With increasing magnetic field $B$, the Fermi energy decreases and the filling factor $\nu$ lowers. In the absence of e-e interaction (solid curve in Fig. 2), with increasing $B$ a step decrease in $E_{s}$ can be observed when $\nu$ is around an even integer number and, otherwise, $E_{s}$ decreases slightly. In sharp contrast, in the pres-

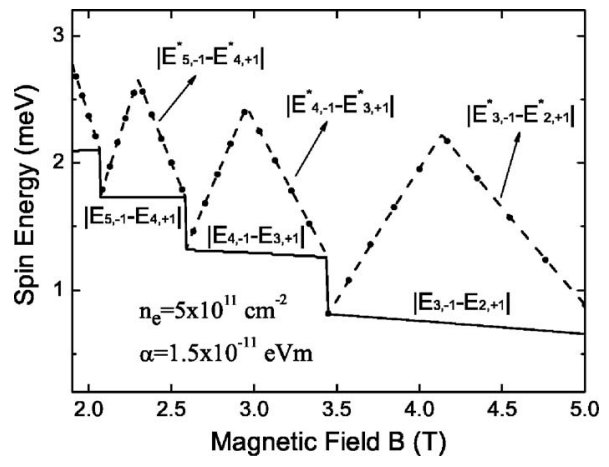

FIG. 2. Spin energy around the Fermi level as a function of magnetic field at the fixed Rashba parameter $\alpha$ and electron density $n_{e}$ as indicated. Here the spin energy is defined as $E_{s}=\left|E_{N,+1}-E_{N+1,-1}\right|$ or $E_{s}^{*}=\left|E_{N+1}^{*}-E_{N+1,-1}^{*}\right|$ for the case of the absence (solid curve) or presence of e-e interaction (dotted curve induced by intra-LL scattering alone and dashed curve induced by the sum of intra- and inter-LL scattering events). 
ence of exchange interaction (dashed and dotted curves in Fig. 2), with increasing magnetic field $B$ the spin-energy $E_{s}^{*}$ first increases when $2 n-1<\nu<2 n$ (with $n>1$ being an integer number), then has a peak value at $\nu=2 n-1$, then decreases when $2 n-2<\nu<2 n-1$, and has a step decrease at $\nu=2 n-2$. Generally, $E_{s}^{*}$ is much larger than $E_{s}$ especially at $\nu \sim 2 n+1 . \nu \sim 2 n$ is a case in which both spin-up and -down states are occupied by electrons. In such a case, the weakest exchange interaction occurs and the step decrease in the spin energy can take place. On the other hand, $\nu \sim 2 n+1$ is a case in which the Fermi level is in between the spin-up and spindown states so that the strongest exchange effect can be observed and the peak value of the spin energy can be achieved. This picture is very similar to that for a $2 \mathrm{DEG}$ in the absence of the RSOI, where the oscillations of the exchange-induced $g$-factor with the magnetic field can be observed both experimentally and theoretically. ${ }^{10}$ Furthermore, the obtained results (dashed and dotted curves in Fig. 2) show that the exchange interaction is mainly achieved via intra-LL scattering [i.e., $N^{\prime}=N$ in Eq. (4)], similar to a spindegenerate $2 \mathrm{DEG}$. $^{10}$

In this study, we have considered that the exchange scattering is induced only by e-e interaction through the Coulomb potential. It is known that the exchange interaction can also be achieved via electron interactions with scattering centers such as impurities and phonons. ${ }^{10}$ The inclusion of the self-energy induced by electron-impurity and electronphonon interaction needs considerable further analytical and numerical calculations, and we do not attempt this in the present study. However, from the fact that the electron interaction with scattering mechanisms is also an important source for exchange-enhanced spin-splitting in a 2 DEG without the RSOI, it can be expected that the exchange interaction via electronic scattering with impurities and phonons can enhance further the spin-energy in a 2DEG in the presence of the RSOI.

\section{CONCLUDING REMARKS}

The results obtained from this theoretical work have demonstrated that a many-body effect such as the exchange interaction induced by e-e interaction is an important source for the enhancement of the spin-splitting in a 2DEG in the presence of the RSOI and quantizing magnetic fields. This can interpret at least partly why the values of the Rashba parameter determined from high-field magnetotransport measurements are much larger than those obtained theoretically from the $\mathbf{k} \cdot \mathbf{p}$ calculation ${ }^{4}$ (single-particle approach) and the self-consistent calculation on the basis of the $\mathbf{k} \cdot \mathbf{p}$ results ${ }^{5}$ (exchange interaction in the $x y$ plane is not included). We hope this work can achieve an in-depth understanding of the physics mechanisms responsible for the enhanced spinsplitting observed experimentally in InGaAs-based spintronic systems.

\section{ACKNOWLEDGMENTS}

This work was supported in part by the Australian Research Council, the NSF of China (Grant No. 10664006), the Knowledge Innovation Program of the Chinese Academy of Sciences, and the NSF of Jiangsu Higher Education Institutions (Grant No. 04KJB510066).

${ }^{1}$ See, e.g., R. Winkler, Spin-Orbit Coupling Effects in $2 D$ Electron and Hole Systems (Springer, Berlin, 2003).

${ }^{2}$ For a recent review, see, e.g., W. Zawadzki and P. Pfeffer, Semicond. Sci. Technol. 19, R1 (2004).

${ }^{3}$ See, e.g., D. Grundler, Phys. Rev. Lett. 84, 6074 (2000).

${ }^{4}$ Th. Schapers, G. Engels, T. Klocke, M. Hollfelder, and H. Luth, J. Appl. Phys. 83, 4324 (1998).

${ }^{5}$ Y. P. Lin, T. Koga, and J. Nitta, Phys. Rev. B 71, 045328 (2005).

${ }^{6}$ T. Nagao, T. Hildebrandt, M. Henzler, and S. Hasegawa, Phys. Rev. Lett. 86, 5747 (2001).

${ }^{7}$ See, e.g., A. Manolescu and R. R. Gerhardts, Phys. Rev. B 51, 1703 (1995).

${ }^{8}$ G. H. Chen and M. E. Raikh, Phys. Rev. B 60, 4826 (1999).

${ }^{9}$ A. Manolescu and R. R. Gerhardts, Phys. Rev. B 51, 1703 (1995).

${ }^{10}$ See, e.g., W. Xu, P. Vasilopoulos, M. P. Das, and F. M. Peeters, J. Phys.: Condens. Matter 7, 4419 (1995).

${ }^{11}$ S. Q. Shen, M. Ma, X. C. Xie, and F. C. Zhang, Phys. Rev. Lett. 92, 256603 (2004).

${ }^{12}$ T. Ando, A. B. Fowler, and F. Stern, Rev. Mod. Phys. 54, 437 (1982).

${ }^{13}$ See, e.g., R. G. Mattuck, A Guide to Feynman Diagrams in the ManyBody Problem (McGraw-Hill, New York, 1976).

${ }^{14}$ J. T. Devreese, A. B. Kunz, and T. C. Collins, Elementary Excitations in Solid, Molecules and Atoms (Plenum, New York, 1974). 